\title{
Accurate determination of gain and radiation patterns by radar cross-section measurements
}

\section{Appel-Hansen, Jørgen}

\section{Published in:}

I E E E Transactions on Antennas and Propagation

Publication date:

1979

Document Version

Publisher's PDF, also known as Version of record

Link back to DTU Orbit

Citation (APA):

Appel-Hansen, J. (1979). Accurate determination of gain and radiation patterns by radar cross-section measurements. I E E E Transactions on Antennas and Propagation, 27(5), 640-646.

\section{General rights}

Copyright and moral rights for the publications made accessible in the public portal are retained by the authors and/or other copyright owners and it is a condition of accessing publications that users recognise and abide by the legal requirements associated with these rights.

- Users may download and print one copy of any publication from the public portal for the purpose of private study or research.

- You may not further distribute the material or use it for any profit-making activity or commercial gain

- You may freely distribute the URL identifying the publication in the public portal

If you believe that this document breaches copyright please contact us providing details, and we will remove access to the work immediately and investigate your claim. 


\title{
Accurate Determination of Gain and Radiation Patterns by Radar Cross-Section Measurements
}

\author{
J $\emptyset$ RGEN APPEL-HANSEN, MEMBER. IEEE
}

\begin{abstract}
Using a two-port network and geometrical interpretation of equations involved in antenna scattering, it can be derived that antenna characteristics may be determined in properly designed scattering measurements. As an alternative to this approach it is shown that measurement procedures for gain and radiation pattern can be developed from simple considerations of the receiving, transmitting, and scattering properties of antennas. The main advantages of the technique are that no gain standard is required and a disturbing feedline to the antenna can be avoided. In addition to this the technique seems to be highly accurate. These general conclusions are well corroborated by experimental data on a standard gain horn. Sources of errors are outlined and compared with sources of errors in conventional techniques.
\end{abstract}

\section{INTRODUCTION}

D UE TO THE costs of satellite experiments and communication links, there is an increasing demand for accurate determination of antenna characteristics, in particular, antenna pattern and gain. Determination of these characteristics by scattering cross-section measurements has been studied previously [1]-[6]. To the author's knowledge Gruber [1] and Sinclair, Jordan, and Vaughan [2] seem to be some of the very first who have reported this type of measurement. King [3] suggested using scattering data as a clue to the mechanism of antennas. McEntee [4] developed a simple technique to measure the absolute values of the scattering aperture and absorption aperture of an antenna with a short-circuited feed. Kennaugh [5] derived the change in the scattering cross section of antennas produced by load variations. Using geometrical interpretation of the equations involved in antenna scattering, Garbacz [6] developed a detailed theoretical background for scattering measurement procedures for the determination of input impedance, gain, and the scattering cross section of an antenna as a function of its load. The present paper is limited to gain and radiation pattern measurements. For this type of measurement an approach other than that of [6] is used. In the present approach procedures are developed from a simple consideration of the receiving, transmitting, and scattering properties of antennas. Thus it has the advantage of avoiding the geometrical interpretations used in [6]. Furthermore, avoiding the use of a two-port network for the measurement situation, it makes use of only the definitions of gain, receiving cross section, and backscattering cross section. It is the purpose of this paper to present the approach and to describe the experiments which were carried out in the Radio Anechoic Chamber of the Technical University of Denmark in order to verify the theory and provide an indication of the accuracy of the developed technique. The types and sources of error in the technique are outlined but not analyzed in detail. However,

Manuscript received July 22, 1977; revised May 1, 1978.

The author is with the Radio Anechoic Chamber, Electromagnetics Institute, Technical University of Denmark, DK-2800 Lyngby, Denmark. by a comparison with the sources of errors in conventional techniques, it is demonstrated that in some cases the RCS technique may have conspicuous advantages. The technique is referred to as the RCS technique since it depends upon measuring the backscattering cross section or the radar cross section of the antenna in short-circuited and matched experiments.

It is a well-known fact that a receiving antenna scatters power in order to receive power from an incident wave [7]. For simple antennas such as small loops and dipoles the received power has the same magnitude as the scattered power. This has also been demonstrated to be approximately valid for half-wave dipoles [8]. However, in general no simple relationship seems to exist between the scattered and the received powers. But it has been demonstrated that for a large class of antennas the scattered power is generally greater than the received power, equality being attained for so-called minimumscattering antennas [9]. The scattered power is distributed in space and the amount of power density scattered in an arbitrary direction is characterized by a scattering cross section in that direction. Furthermore, the variation of the scattered power density with aspect angle depends upon the direction of incidence. In fact, the antenna may be considered as an object on which a current and charge distribution are generated. Thus the receiving antenna is a loaded scatterer for which the scattering cross section can be calculated as outlined in [10]. In case the antenna is not matched to its transmission line, part of the received power is reflected at the load and reradiated. For the present author the cue to the RSC technique was given by imagining a matched antenna load replaced by a short-circuit. Then the total received power would be reradiated in accordance with the radiation properties of the antenna. The reradiated power will interfere with the scattered power, i.e., both reradiated and scattered powers will contribute to the scattering cross section of the short-circuited antenna. By varying the position of the short, the numerical value in decibels, of the ratio between reradiated and scattered powers can be determined. The sign of the ratio may be determined by measuring the scattering cross section in a matched experiment. Following this the scattering cross sections associated with the reradiated power and the scattered power may be assessed by using a reference sphere as in usual RCS measurements [11]. Then a simple computation will give the gain of the antenna in the direction considered. It should be mentioned that the relationship between the scattering cross section and commonly defined antenna parameters for arbitrary loads is known [12], [13]. Therefore, this relationship may be used as an alternative to describe and develop the RCS technique.

In Section II the theoretical background for the experimental procedure is given. As can be imagined, no feedline to the antenna is required in the short-circuited and the matched experiments. This is an advantage since the feedline may 
disturb the measurements drastically [14], and proper reduction of the influence of the cable may require ingenious feeding of the antenna. It should be noted that instead of having a feedline to disturb the antenna properties, they may be influenced by the load and the movable short. However, these components may be minimized and arranged so that they have negligible influence on the antenna properties. It should also be mentioned that the impedance characteristics of the shortcircuit and the matched load may be better and easier to achieve than in conventional techniques.

It should be clarified that in actual antenna systems there is some sort of feeding system. Thus for the final communication link it is of interest to know the gain of the actual system when the antenna is mounted and connected to its feeding system. However, in the design of systems and in many theoretical studies it is of interest to know the gain of the antenna without any additional components connected to its terminals. Furthermore, in the measurement of the gain of antennas placed on spacecrafts it might be convenient to short circuit the antenna instead of having a separate feeding system, as in a standard setup. For the sake of completeness it should be mentioned that the RCS technique may also be used when it is desired to measure the gain of the actual system, where the antenna is mounted and connected to its feeding system. In this case the short is simply placed at the end of the feedline.

Besides having the option of avoiding a feedline to the antenna, another attractive advantage of the method is that no reference standard gain antenna is needed. Although there is a demand for accurately calibrated reference antennas, none commercially available seem to exist. The RCS technique avoids this demand by using a reference sphere to establish the reference level needed in the experiments. Since reference spheres can be manufactured accurately, and their scattering properties can be determined theoretically with a high degree of accuracy, no experiment is required to establish the absolute value of their backscattering cross section.

The experimental setup is described in Section III. This has been used and continuously refined over the past ten years. In the experimental procedure the short is placed in different positions, and for each position the short-circuited antenna is moved. The analysis of the results obtained in this manner will reduce the influence of reflections from the lining of the anechoic chamber and the support on the desired received signal [15].

The results are given in Section IV. In all cases the agreement between the radiation pattern and the gain of an antenna obtained by the RCS technique and various conventional techniques was satisfactory. The author was then encouraged to use it for the determination of the gain of a horn antenna mounted on a relatively large metal plate. A conventional measurement of the gain was difficult due to the mounting plate which caused multiple reflections. These were reduced in the conventional technique by moving the test antenna. The results obtained in this manner agreed within a $0.4-\mathrm{dB}$ discrepancy with the results obtained by using the RCS technique [15]. Another example of using the RCS technique is in the determination of the scattering properties of a rectangular waveguide to be used as a probe in near-field techniques. In addition to this, it has also been demonstrated that the RCS technique can be used to determine the insertion loss of various components. It may also be used in optical fibers where an open-ended fiber may be used as an antenna.

It is expected that all antenna characteristics, including its

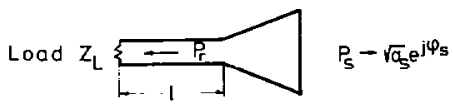

(a)

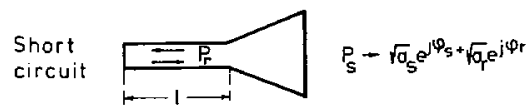

(b)

Fig. 1. RCS technique. (a) Matched antenna. (b) Short-circuited antenna.

impedance and polarization properties, can be measured by properly designed scattering measurements. It is hoped that the present paper contributes to the understanding of the operation of an antenna and helps in the development of new measurement techniques which can be used to determine its characteristics. In future studies special attention has to be given to an evaluation and comparison of different sources of errors in conventional and scattering techniques.

\section{THEORY}

In order to provide a background for the experiments described in the next sections it is convenient to consider in some detail the interaction between a receiving antenna and an incident wave. In Fig. 1 (a) a receiving antenna is shown connected through a lossless transmission line of length $l$ to a conjugate matched load $Z_{L}$. When a wave is incident on the antenna a current distribution is generated on the antenna. This current distribution causes two effects. First, the current distribution radiates power. This power is referred to as the scattered power $P_{s}$. The power $P_{s}$ is scattered in space, and the antenna may be considered as a scattering object with scattering cross section $\sigma_{s}$. The corresponding scattered field intensity is proportional to $\sqrt{\sigma_{s}} e^{j} \phi_{S}$, where $\phi_{s}$ is its phase relative to some arbitrary reference. Second, the current distribution causes a voltage to be generated across the terminals of the load impedance. This results in a power $P_{r}$ which is received or absorbed by the load. Suppose now that the load is replaced by a short circuit as shown in Fig. 1 (b). Then the power $P_{r}$ which propagates along the transmission line toward the short circuit is reflected back to the antenna and reradiated in space. Therefore, for the short-circuited antenna we will refer to $P_{r}$ as the reradiated power. Besides having this reradiation the short-circuited antenna is assumed to scatter the same amount of power $P_{s}$ as when it is connected to $Z_{L}$. In order to consider the interference between the scattered and reradiated powers, it is convenient to define a scattering cross section $\sigma_{r}$ which is due to $P_{r}$, i.e., $\sigma_{r}$ is the scattering cross section which the short-circuited antenna would have in case only the reradiated power $P_{r}$ existed. The field intensity corresponding to $P_{r}$ is proportional to $\sqrt{\sigma_{r}} e^{j \phi_{r}}$, where $\phi_{r}$ is its phase. From this discussion it appears that the total scattered field is the sum of two components, one proportional to $\sqrt{\sigma_{s}} e^{j \phi_{s}}$ and the other proportional to $\sqrt{\sigma_{r}} e^{j \phi_{r}}$. The parameters $\sigma_{s}, \sigma_{r}, \phi_{s}$, and $\phi_{r}$ depend upon the direction considered. In order to treat the measurement problem in general it is to be noted that the power which is reradiated when the antenna is short-circuited has the same magnitude as the power which is received when the antenna is connected to the load $Z_{L}$. Furthermore, it is assumed above that the scattered power has the same distribution in space for both cases of matched and short-circuited antennas, i.e., when the load $Z_{L}$ is replaced by the short the 
current distribution which causes the scattered power is not changed. This requires that in the actual experiments both load and short must have the same outer surface and that the same single mode must be propagating inside the waveguide components.

In order to derive the basic relation which constitutes the background for the experimental procedure, suppose that a plane wave is incident from an arbitrary direction. Let the incident power density at the antenna be $S_{i}$. Then the received power $P_{r}$ is given by

$$
P_{r}=S_{i} A
$$

where $A$ is the receiving cross section of the antenna in the direction considered. When the antenna is short-circuited this power is reradiated in accordance with the transmitting properties of the antenna. At a distance $R$ in the direction back in the direction of incidence, the reradiated power density is

$$
S_{r}=\frac{S_{i} A G}{4 \pi R^{2}},
$$

where $G$ is the gain in the direction considered. The backscattering cross section $\sigma_{r}$ of the antenna associated with the reradiated power is found from

$$
\sigma_{r}=\lim _{R \rightarrow \infty} 4 \pi R^{2} \frac{S_{r}}{S_{i}}
$$

which simplifies to

$$
\sigma_{\mathrm{r}}=A G
$$

which is the basic relation. It should be mentioned that a similar relation was derived in a laboratory report by McEntee [4].

As mentioned above the scattered power and the reradiated power contribute to the total backscattering cross section of the antenna connected to the short circuit. Usually no simple relation exists between the scattered and the reradiated powers. However, one important exception to this exists if the power scattered by the open-circuited antenna is much less than that scattered by the matched antenna. In this case $\sigma_{s}$ is approximately equal to $\sigma_{r}$, i.e., $\sigma_{s}$ is also equal to the product of the gain and receiving cross section [8]. It is interesting to note that (4) is valid in general, but usually no simple relation seems to exist for $\sigma_{s}$. In the Appendix it is shown that (4) can be derived from the equations presented in [6].

In the actual measurements the position of the short is changed. This means that the phase $\phi_{r}$ of the reradiated field is varied. Thus it is possible to measure the in-phase and out-ofphase interference between the scattered and reradiated field. At the maximum and minimum of the interference curve the backscattering cross sections $\sigma_{\max }$ and $\sigma_{\min }$, respectively, are measured. Hence

$$
\sigma_{\max }=\left(\sqrt{\sigma_{s}}+\sqrt{\sigma_{r}}\right)^{2}
$$

and

$$
\sigma_{\min }=\left({\sqrt{\sigma_{s}}}-{\sqrt{\sigma_{r}}}^{2}\right.
$$

The absolute values of $\sigma_{\max }$ and $\sigma_{\min }$ are determined by using reference spheres to calibrate the setup as in conventional RCS measurements. Initially the magnitudes of $\sigma_{r}$ and $\sigma_{s}$ relative to each other are not known. Therefore, from (5) and (6) two cross sections are determined, one belonging to $\sigma_{s}$ and the other belonging to $\sigma_{r}$. In order to solve the ambiguity, $\sigma_{s}$ can be determined by measuring the scattering cross section of the antenna matched to the transmission line as shown in Fig. 1 (a).

After the determination of $\sigma_{r}$ the gain is found from the equation

$$
G=\frac{\sqrt{4 \pi}}{\lambda} \sqrt{\sigma_{r}}
$$

which is derived from (4) by making use of the relation

$$
A=\frac{\lambda^{2}}{4 \pi} G
$$

In the actual experiments the signal levels may be recorded in decibels, and it is convenient to use (7) in a decibel scale. It will then become

$$
G(\mathrm{~dB})=-9.275+10 \log f(\mathrm{GHz})+\frac{1}{2} \sigma_{r}\left(\mathrm{~dB} / \mathrm{cm}^{2}\right)
$$

where $G$ is calculated in decibels from the measured values of $\sigma_{r}$ in $\mathrm{dB} / \mathrm{cm}^{2}$ at a frequency $f$ in $\mathrm{GHz}$. The frequency is given in $\mathrm{GHz}$ because the above given theory is verified in the microwave region as described in the next section. From (9) it is to be noted that an error of $\pm \Delta \mathrm{dB}$ in the determination of $\sigma_{r}$ gives an error of only $\pm \frac{1}{2} \Delta \mathrm{dB}$ in the determination of $G$. This is due to the fact that the short-circuited antenna receives and reradiates the same amount of power.

It was mentioned above that $\sigma_{r}$ could not be determined uniquely from the interference curve, since it was not initially known which one of the cross sections, $\sigma_{r}$ or $\sigma_{s}$, is the larger one. The ambiguity was resolved by carrying out a matched experiment. Other procedures can be developed from the following consideration of the short-circuited experiment of Fig. 1 (b). Here the total field is the sum of the scattered and the reradiated fields. In case a short-circuited experiment could be repeated under such conditions that either $\sigma_{s}$ or $\sigma_{T}$ were changed, the ambiguity could be resolved in the following manner. The analysis of the interference curve of the repeated experiment would again give two cross sections. The two cross sections, one from the original short-circuited experiment and the other from the repeated experiment, that are closest to each other may be identified as the cross section which was not changed. Smaller differences may be attributed to imperfections in the experiments and normal experimental inaccuracies. The reader can imagine that in some experiments $\sigma_{r}$ and $\sigma_{s}$ may be so close to each other that additional experiments have to be carried out.

In some cases it is not difficult to change either $\sigma_{s}$ or $\sigma_{r}$ and carry out the above analysis. In fact, the cross section $\sigma_{s}$ was changed in experiments with a horn antenna. A reflecting plate was mounted behind the horn aperture. This resulted in a considerable change in the scattering properties without any appreciable change in the receiving properties. In other experiments the cross section due to reradiation was changed simply by inserting an attenuator between the antenna and the short- 
circuit. Since the recieved power was attenuated both when it propagated toward the short and after it was reflected by the short, the measured value of $\sigma_{r}$ was decreased by a factor equal to twice the insertion loss of the attenuator relative to the value of $\sigma_{r}$ measured without the attenuator inserted. In the actual experiment, within a few tenths of a decibel variation, the measured values of $\sigma_{r}$ agreed with variation in the calibrated attenuator. As expected from the theory above, $\sigma_{s}$ was measured to be independent of the position of the attenuator. From this it is also understood that the RCS technique may be used to determine the insertion loss of a component by measuring $\sigma_{r}$ before and after the insertion of an unknown component. Without going into details it should be mentioned that the ambiguity may also be resolved by measuring the phase variation of the total scattered field when the position of the short is varied.

Additional experiments as described above may be saved in some cases. The ambiguity may be resolved simply by using (9) to calculate two values of $G$ from the two cross sections obtained from a short-circuited experiment. One of the gain values belongs to the reradiation, i.e., it is equal to the normal antenna gain, and the other gain value belongs to the scattering properties of the antenna. Knowing approximately the gain of the antenna the engineer may decide which one of the two gain values is representing the antenna gain. As an alternative to knowing the gain of the antenna, for example in the boresight direction, the variation of the gain with frequency or aspect angle may be known. This information can be utilized to resolve the ambiguity simply by carrying out several shortcircuited experiments at different frequencies or different aspect angles.

It is interesting to note that by calculating two gain values and identifying one as the normal antenna gain, the other gain value will represent the scattering properties of the antenna. Thus, besides determining the receiving and transmitting properties of antennas, the RCS technique at the same time provides information on the scattering properties of the antennas under test.

\section{EXPERIMENTAL SETUP}

The theory is verified by using the experimental setup shown in Fig. 2. It is a nearly monostatic radar arranged in a radio anechoic chamber lined with 2 -m high absorbers. The details of the operation and an evaluation of the setup are given in [15]. The major features will be given in the following. Part of a frequency-stabilized signal is guided through a uniline, cross coupler, and attenuator to a transmitting horn and radiated towards the test object. Another part of the stabilized signal is guided through the cross couplers to the mixer and receiver when the switch is in the proper position. This cross-coupled signal may be adjusted in phase and amplitude by the ten-screw tuner. The scattering from the test object is absorbed by the receiving horn and is guided to the receiver. The cross-coupled signal and the scattering from the test object was received together with the scattering from the lining of the anechoic chamber (the background signal), the scattering from the support for the antenna under test, the coupling between the two horns, and other leakage signals.

The measurements are carried out by using the so-called hybrid technique, i.e., a combination of the conventional cancellation and the overriding techniques [15]. In the hybrid technique the received signal is tuned to a minimum by means of the ten-screw tuner. This initial step is carried out

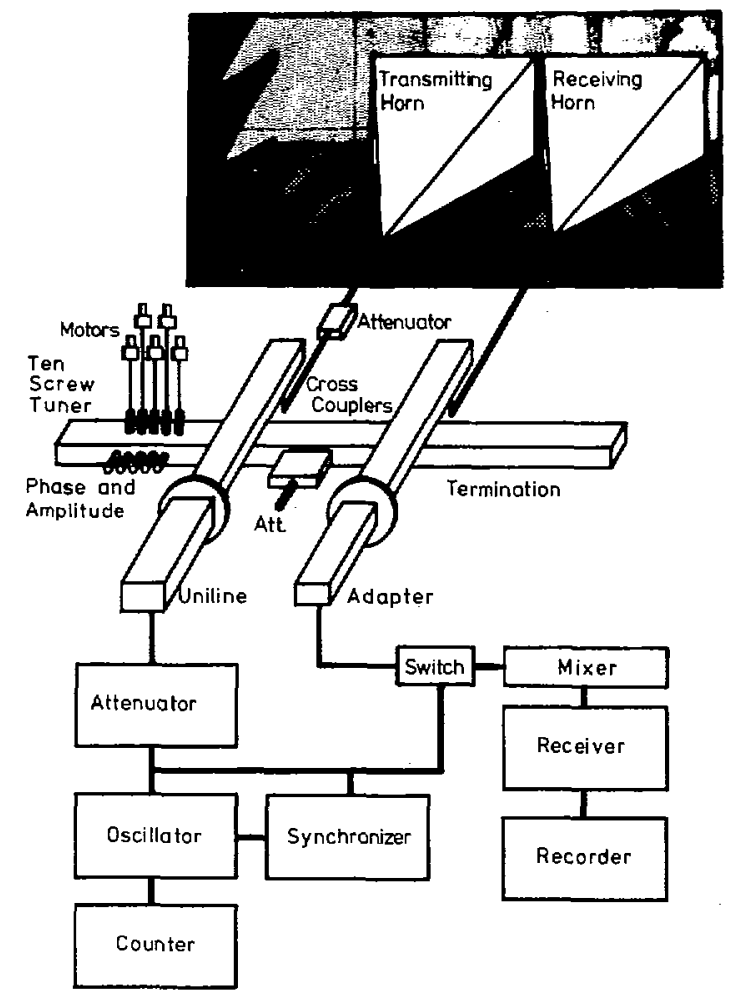

Fig. 2. Experimental setup.

before mounting the test object on its support, which can be moved about. After insertion of the test object the support is moved back and forth. During the movements a curve giving the interference between the combined signal from the test object and the support and the sum of all the remaining received signals, the so-called setup signal, is recorded. It is the influence of the setup signal which is attempted to be minimized in the initial step. The setup signal is usually not negligible because the system cannot be tuned out completely in the initial step. In addition to this the support is part of the tuned signal, and insertion of the test object changes the background signal. The interference curves recorded during the movements of the test object are often referred to as the standing wave curves owing to their similarities with the normal voltage standing-wave ratio (VSWR) curves.

In the present verification it turned out that it was possible to carry out the experiments in the lower case overriding mode of operation, where the setup signal is less than the signal from the test objects. These were a reference sphere of radius 4.5 in and a standard gain antenna connected either to a short circuit or a match load in accordance with the theory presented in the previous section. The reference sphere was used to establish a reference level for the results. Its theoretical radar cross section in $\mathrm{dB} / \mathrm{cm}^{2}$ was assigned to an average recorded level determined in the following manner. In order to partially eliminate the influence of the support on the reference level the sphere was measured in two positions separated by a distance of $2.5 \mathrm{~cm}$, i.e., about a quarter of a wavelength in the frequency band considered in the present work. This was in fact carried out before and after the antenna under test was measured. Comparing the results it could be observed whether the apparatus had been reasonably stable during the measurements. If this were the case an average of the four measured values for the sphere was used to represent the reference level. 
The reference level obtained by using a sphere is only needed in case the gain of the antenna is to be measured. When radiation patterns are to be measured, a pattern level in a certain direction, for example boresight, can be chosen as a reference level.

The RCS technique in its present form requires that for each aspect angle of the antenna under test, the position of the short has to be varied. Furthermore, for each position of the short, the antenna is moved. This movement is not required by the RCS technique itself, but it is carried out in order to increase the measurement accuracy. In conventional antenna measurements accuracy may also be increased by moving the antenna under test [17]. However, the present setup still has the disadvantage of being time-consuming because results can be obtained only at discrete aspect angles. However, by automation of the equipment and digitizing of data, the most tedious aspects of the technique may be avoided. In fact, in [2] it is demonstrated that by modulating a load connected to the antenna radiation patterns may be easily recorded. In this connection it should be mentioned that having developed an RCS setup, antenna measurements as well as ordinary RCS measurements can be carried out with the same setup. This may in some cases save the time usually required to change the instrumentation from one type of experiment to the other.

\section{RESULTS}

After several introductory experiments, which showed a surprising degree of accuracy, i.e., often within $0.1 \mathrm{~dB}$ of the expected value, it was decided to measure 1) pattern levels at several aspect angles, and 2) the gain as a function of frequency. The antenna selected was a pyramidal standard gain horn with a gain calibration curve supplied by the manufacturer and an $H$-plane pattern measured some years ago in the Radio Anechoic Chamber.

In Fig. 3 the $H$-plane pattern is shown. The solid line curve is measured by using a conventional pattern recording technique. The dots in the circles show the pattern levels measured at several aspect angles by the RCS technique. As usual, the maximum level was denoted $0 \mathrm{~dB}$ in both techniques. In order to compensate for misalignment of the antennas during measuements, the pattern expected from the RCS measurements was adjusted in angle to fit the normal pattern. It should be clarified that compensation for misalignment is not required by the RCS technique itself. It was done in order to compare the results obtained in two different experiments where the positioning of the antenna under test was made with different degrees of accuracy. In short, the requirements of the mechanical positioning of the antennas in the RCS technique are the same as in conventional techniques.

The pattern levels in the RCS technique were obtained by using the level in the boresight direction as a.reference. From measurements of several levels close to the boresight it turned out that although the RCS measurements were carried out at discrete angles and by analyzing several standing wave curves, the RCS technique has a resolution of better than $0.1 \mathrm{~dB}$. The largest discrepancy between the two results is observed at low pattern levels. This is supposed to be due mainly to the influence of the reflections from the lining of the anechoic chamber on the conventionally recorded patterns. It is at particularly low pattern levels that the reflections cause the larger inaccuracies. In fact, these inaccuracies are minimized in the present RCS technique by moving the antenna and recording standing wave curves.

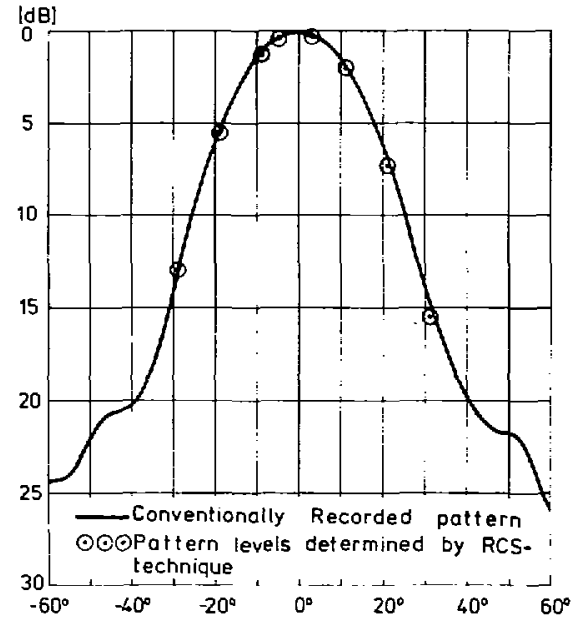

Fig. 3. Antenna radiation pattern.

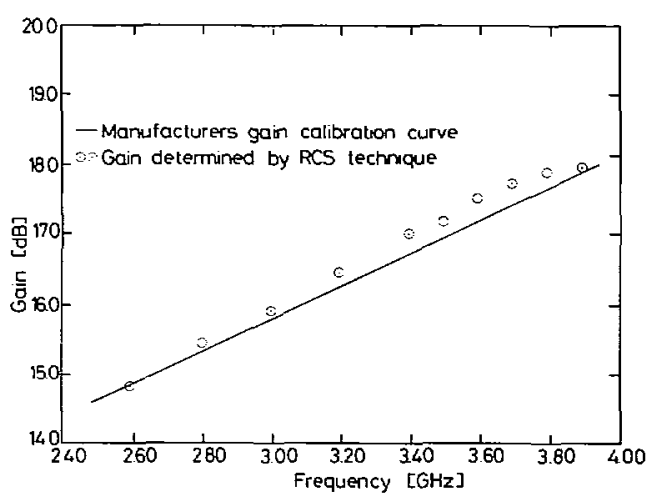

Fig. 4. Gain as a function of frequency.

In Fig. 4 the gain of the horn antenna is shown as a function of frequency. The solid line is a copy of the actual curve supplied by the manufacturer of the horn. The dotted circles indicate the values obtained by the RCS technique. It is seen that the RCS technique gives results which do not make a straight line as the results given by the manufacturer do. Furthermore, the results seem to be about $0.2 \mathrm{~dB}$ too high. These discrepancies may be due to many sources of error, owing to the fact that the two results are from two completely different experiments. A full analysis is not given here, since detailed information on the manufacturers experimental setup and procedure was not available.

The major sources of error in the present experimental setup are as follows:

1) determination of the reference level of the 4.5-in standard sphere;

2) nonlinearity in the receiving system;

3) variation in the power level, receiver sensitivity, and frequency;

4) uncertainty in the reading of the standing wave curves;

5) uncertainty in the determination of $\sigma_{\max }$ and $\sigma_{\min }$ from the measured interference pattern between the scattered and reradiated signals.

All of these sources of error can easily be improved by refining the technique. In fact, the source of error 5) has already been improved by developing a computer program using curve fitting [18]. 
Other sources of error are as follows:

6) influence of extraneous reflections from the test range;

7) impedance mismatch loss;

8) loss in the short-circuiting component;

9) the small inherent bistatic angle in the nearly monostatic radar used in the present experiments (this error can be eliminated by using a monostatic radar);

10) the technique does not alleviate the problems of measuring in the "near-field" of the antenna under test [19];

11) positioning the antenna and the reference sphere.

Thus the RCS technique includes many errors including some which are hard to identify. However, similar errors are present in conventional antenna measurement techniques. In fact, for the conventional techniques all of the above-listed errors are valid except errors 1),5), and 11), which are pertinent to the RCS technique. But for conventional techniques there are also sources of errors which are not pertinent to the RCS technique. For example, in an experiment where the gain of the antenna under test is determined by comparison with a standard gain antenna, some of the sources are

a) determination of the reference level of the standard gain antenna,

b) positioning of the antenna and the standard gain antenna,

c) influence of feeding system,

d) the requirement of an accurately known standard gain antenna.

Errors a) and b) correspond to errors 1) and 11). For investigations where the gain of the antenna without a feedline are required, error c) is lacking in the RCS technique. The error corresponding to d) in the $\mathrm{RCS}$ technique is the knowledge of the radar cross section of a reference sphere. However, this error is negligible because the radar cross section of spheres can be calculated with a high degree of accuracy.

It has not been within the scope of the present investigation to undertake a detailed analysis of different errors. However, error 11), which is related to the assessment of the distance from the radar unit to the test object, has particularly puzzled the author. In the actual measurement setup the distances from the aperture of the radar antennas to the front surface of the reference sphere and the aperture of the antenna under test are $929.0 \mathrm{~cm}$ and $913.5 \mathrm{~cm}$, respectively. In the case where these distances are used to represent the distances to the sphere and antenna under test, then a correction is required of the reference level determined by the sphere. Using the radar equation it is found that the measured gain yalues should be reduced $0.15 \mathrm{~dB}$. In the case where this reduction of the actual results in Fig. 4 is carried out, an improved agreement between the manufacturers and the present results is obtained. In fact, an average discrepancy between the results in Fig. 4 is found to be $0.20 \mathrm{~dB}$. It might seem reasonable to make the reduction of $0.15 \mathrm{~dB}$, which is due to the difference in the above-given distances, before plotting the results in Fig. 4 obtained by the RCS technique. However, the author wishes to carry out a more detailed anlaysis of the sources of error before claiming that the RCS technqiue is in general accurate within a few hundredths of a decibel. At present the allotment of measurement time and other resources have come to an end.

\section{CONCLUSION}

From simple considerations of the receiving, transmitting, and scattering properties of antennas, it has been shown that an RCS technique for determining the radiation pattern and gain of antennas can be developed. The technique has been verified by measuring the pattern of an antenna and the variation of its gain with frequency. The results obtained in the first experiments agreed within a few tenths of a decibel with the results obtained by conventional techniques. Since different sources of error influence the conventional and the new results, it is expected that an error analysis will show complete agreement. Furthermore, by refining the RCS technique it may provide results which are more accurate than those obtained by using conventional techniques. This is due mainly to the fact that no feedline or reference antennas are needed in the RCS technique.

\section{APPENDIX}

In [6] it is shown that

$$
G_{r} G_{t}=\frac{\pi}{p_{r} p_{t}}\left|\frac{1}{\lambda}\left(\sqrt{\sigma_{\max }} \pm \sqrt{\sigma_{\min }}\right)\right|^{2},
$$

where $\lambda$ is the wavelength, $p_{r}$ and $p_{t}$ are the polarization mismatch factors, $G_{r}$ and $G_{t}$ are the power gains of antenna under test in the direction of the receiver and transmitter, respectively, and $\sigma_{\max }$ and $\sigma_{\min }$ are the maximum and minimum scattering cross sections measured when a reactive load is connected to the antenna under test and varied. The cross sections $\sigma_{\max }$ and $\sigma_{\min }$ are obtained when the scattered and reradiated fields are in and out of phase, respectively. The use of the positive and negative signs depends upon the magnitude of a constant C. Without going into detail let us suppose that the negative sign is valid. This implies that the scattered field is larger in magnitude than the reradiated field. Furthermore, suppose that the receiver and the transmitter are placed in the same direction, i.e., the backscattering cross section is measured, and that the setup is polarization-matched to the antenna under test $\left(p_{r}=p_{t}=1\right)$, then

$$
G^{2}=\frac{\pi}{\lambda^{2}}\left|\sqrt{\sigma_{\max }}-\sqrt{\sigma_{\min }}\right|^{2}
$$

where $G$ is the gain of the antenna in the direction considered. Using the terminology of the present paper, we have

$$
\sqrt{\sigma_{\max }}=\sqrt{\sigma_{s}}+\sqrt{\sigma_{r}}
$$

and

$$
\sqrt{\sigma_{\min }}=\sqrt{\sigma_{s}}-\sqrt{\sigma_{r}}
$$

Introducing relations (A3) and (A4) into (A2), the basic relation

$$
\sigma_{r}=A G
$$

is obtained by making use of

$$
A=\frac{\lambda^{2}}{4 \pi} G
$$

where $A$ is the receiving cross section of the antenna. 


\section{ACKNOWLEDGMENT}

The author is grateful to his colleagues for stimulating discussions and to P. Laugesen, V. Maegaard, R. W. Perriard, and $\mathbf{J}$. Warych for their help during the measurements. Thanks are also due to L. Shafai for commenting on the manuscript and to Henry Galle Stech for bringing previous works in the area to the author's attention.

\section{REFERENCES}

[1] J. R. Gruber, "Investigation of the method of measuring antenna patterns by utilizing the reradiated electromagnetic fields," master's thesis, Dep. Elec. Eng., Ohio State Univ., Columbus, 1946.

[2] 'G. Sinclair, E. C. Jordan, and E. W. Vaughan, "Measurement of aircraft-antenna pattern using models," Proc. IRE, vol. 35, pp. 1451-1467, Dec. 1947.

[3] D. D. King, "Measurement and interpretation of antenna scattering," Proc. IRE, vol. 37, pp. 770-777, July 1949.

[4] J. A. McEntee, "A technique for measuring the scattering aperture and absorption aperture of an antenna," Antenna Lab., Ohio State Univ., Columbus, Rep. no. 612-14, Jan. 1957.

[5] E. M. Kennaugh, "The echoing area of antennas," Antenna Lab., Ohio State Univ., Columbus, Rep. no. 601-14, Dec. 1957.

[6] R. J. Garbacz, "Determination of antenna parameters by scattering cross-section measurements," Proc. IEE, vol. 111, no. 10, Oct. 1964.

[7] S. Silver, Microwave Antenna Theory and Design. MIT Radiation Lab. Ser., vol. 12. New York: McGraw-Hill, 1949, pp. 41-44.

[8] J. Appel-Hansen, "A Van Atta reflector consisting of half-wave dipoles," IEEE Trans. Antennas Propagat., vol. AP-14, no. 6, pp. 694-700, Nov. 1966.

[9] W. K. Kahn and H. Kurss, "Minimum-scattering antennas," IEEE Trans. Antennas Propagat., vol. AP-13, pp. 671-675, Sept. 1965.

[10] R. F. Harrington, Field Computation by Moment Methods. New York: Macmillan 1968, pp. 115-120.

[11] J. W. Crispin, Jr., and K. M. Siegel, Methods of Radar CrossSection Analysis, New York: Academic, 1968, p. 355.

[12] R. F. Harrington, "Theory of loaded scatterers," Proc. IEE, vol. 111 , no. 4, pp. 617-623, Apr. 1964.

[13] R. E. Collin and F. J. Zucker, Antenna Theory, Inter-Univ. Elec. Series, vol. 7. New York: McGraw-Hill, 1969, pp. 123133.

[14] J. Appel-Hansen and O. Rieck Sфrensen, "Influence of feed line on antenna gain," IX Nat. Conv. on Radio Science and the
Nordic Antenna Seminar, URSI Finnish Nat. Comm., Otaniemi, Finland, Conf. Digest, pp. 56-59, Oct. 27-29, 1976.

[15] J. Appel-Hansen, "Precision measurement of backscattering cross-sections as a function of frequency," IEEE Trans. Instrum. Meas., vol. IM-25, no. 4, pp. 363-370, Dec. 1976.

[16] J. Appel-Hansen, "Gain of horn antenna determined from two short-circuited experiments using the RCS-technique," Electromagn. Inst., Tech. Univ. Denmark, Denmark, Rep. IR 128, July 1977.

[17] J. Appel-Hansen, "Reflectivity level of radio anechoic chambers," IEEE Trans. Antennas Propagat. (Special Issue on Antenna Measurements), vol. AP-21, no. 4, pp. 490-498, July 1973.

[18] S. Str $\phi$ h, "Computerprogram for curve fitting of standing wave curves in RCS-techniques," (in Danish) Electromagn. Inst., Tech. Univ, of Denmark Denmark, Rep. IR 133, Jan. 1978.

[19] R. G. Kouyoumjian and L. Petersen, "Range requirement in radar cross-section measurements," Proc. IEEE, vol. 53, pp. 920-928, Aug. 1965.

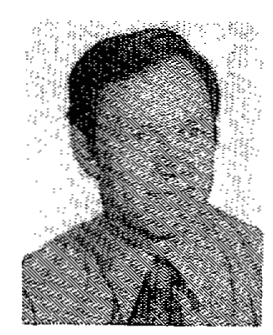

Jørgen Appel-Hansen (M'60) was born in Farendløse, Denmark, on March 13,1937 . He received the M.Sc. degree in electrical engineering in 1962 from the Technical University of Denmark, Lyngby. He received the Ph.D. degree in 1966 from the Laboratory of Electromagnetic Theory, now the Electromagnetics Institute, Technical University of Denmark.

From 1962 to 1964 he was in the Royal Danish Army. Part of the time was spent as a Teaching Associate at the Physics Laboratory II, Technical University of Denmark, Lyngby. In 1967 he was a Visiting Research Associate at the University of Michigan, Ann Arbor. From 1968 to 1978 he worked as head of the Radio Anechoic Chamber, Electromagnetics Institute, Technical University of Denmark. He is currently an Associate Professor at the Electromagnetics Institute. He is also a Consultant in design of anechoic chambers, test ranges, their instrumentation, and implementation of measurement procedures. $\mathrm{He}$ has been engaged in research on retrodirective reflectors, directive loop array antennas, and measuring methods for radio anechioic chambers. $\mathrm{He}$ has organized several international postgraduate courses in measurements in radio anechoic chambers. In 1972 he gave a postgraduate course in antenna measurements at the National Physical Laboratory, New Delhi, India. In February, 1977 he visited the University of Baghdad, Iraq. In May, 1978 he was a Visiting Professor at Universitá di Napoli, Italy. 